UCSD/PTH 97-29

hep-th/9709161

\title{
't Hooft Conditions in Supersymmetric Dual Theories
}

\author{
Gustavo Dotti \\ Department of Physics, University of California at San Diego, \\ 9500 Gilman Drive, La Jolla, CA 92093-0319
}

(September 1997)

\begin{abstract}
The matching of global anomalies of a supersymmetric gauge theory and its dual is seen to follow from similarities in their classical chiral rings. These similarities provide a formula for the dimension of the dual gauge group. As examples we derive 't Hooft consistency conditions for the duals of supersymmetric QCD and $S U(N)$ theories with matter in the adjoint, and obtain the dimension of the dual groups.
\end{abstract}


One important constraint on the moduli space of vacua of supersymmetric gauge theories [1] is that the massless fermions in the low energy theory should have the same flavor anomalies as the fundamental fields, i.e. the 't Hooft consistency conditions should be satisfied [2]. These conditions are used as a test on the spectrum of massless fermions, usually obtained from symmetry arguments and renormalization group flows. Two types of theories have been found: for type I theories the classical moduli space $\mathcal{M}_{\mathrm{cl}}$ or a suitable quantum modified version of it satisfies 't Hooft consistency conditions at every point; type II theories fail to satisfy these conditions at some points of $\mathcal{M}_{\mathrm{cl}}$, and their quantum moduli space cannot just be a quantum modification of $\mathcal{M}_{\mathrm{cl}}$. It is believed that the IR sector of these theories at those points corresponds to a dual theory [3]. The dual theory has a different gauge group and matter content, but the same flavor symmetry group. As an example, consider supersymmetric QCD with $N_{F}$ flavors. When $N_{F}<N$ there is no supersymmetric vacuum in the quantum theory [⿶. When $N_{F} \geq N, \mathcal{M}_{\mathrm{cl}}$ is described by mesons and baryons, which are gauge invariant polynomials in the microcopic fields, subject to some algebraic constraints. On general grounds it is shown that only the $N_{F}=N$ theories admit quantum deformations of the classical constraints. In fact, both $\mathcal{M}_{\mathrm{cl}}$ in $N_{F}=N+1$ theories and a quantum deformation of $\mathcal{M}_{\mathrm{cl}}$ in the $N_{F}=N$ case describe correctly the IR sector of these theories, these are type I theories [四]. However, the origin of $\mathcal{M}_{\mathrm{cl}}$ in $N_{F} \geq N+2$ theories fails 't Hooft's consistency test and is believed to correspond to a dual theory [3]. Recently [5,6], a mechanism responsible for flavor anomaly matching in type I theories was found and used to predict when a theory belongs to this group, avoiding explicit calculation of anomalies. Anomaly matching in s-confining theories [7] (such as QCD with $N_{F}=N+1$ ) and those obtained from them by integrating out matter fields (such as QCD with $N_{F}=N$ ), which have a quantum modified moduli space, follows from the results in [5]. In this letter we explore type II theories. The duality hypothesis in type II theories is supported by a number of consistency checks, of which the matching of global anomalies between both theories is believed to be a particularly stringent one. We will show that this matching follows from a sequence of applications of the results in [5,6] and the relation between the classical moduli space of both theories, intimately related to their classical chiral rings [6].

We first review the notation and state the results we need from [5], a complete proof of them, together with a discussion on the classical moduli space from an algebraic geometry perspective can be found in [6]: $\phi^{i}, i=1, \ldots, d_{U}$ is a point in the vector space $U$ of constant chiral field configurations of the UV theory, $G$ is the complexification of the gauge group $G_{r}$ of the theory, and $G \phi \subseteq U$ is the $G$ orbit of $\phi \in U$. If a tree level invariant superpotential $W(\phi)$ is added to the theory then $U^{W} \subseteq U$ denotes the set of critical points $d W=0$, which contains complete $G$ orbits, as $W$ is $G_{r}$ invariant and holomorphic, therefore $G$ invariant. $V$ is the vector space spanned by a basic set of gauge invariant polynomials $\hat{\phi}^{i}(\phi)$ in $U^{W}$ constructed out of the fundamental fields $\phi^{i}$. The tangent vector space of $U^{W}$ at the point $\phi_{0}$ is denoted $T_{\phi_{0}} U^{W}$. Under the natural isomorphism $T_{\phi_{0}} U \cong U$ we can regard $T_{\phi_{0}} U^{W} \subseteq U$ and expand a tangent vector in coordinates $\delta \phi^{i}$. There is a natural map $\pi: U^{W} \rightarrow V$ by $\phi \rightarrow \hat{\phi}(\phi)$. The image $\mathcal{M}_{\mathrm{cl}}=\pi\left(U^{W}\right)$ is called classical moduli space. The reason is that there is a unique closed orbit in every fiber $\pi^{-1}(\hat{\phi}), \hat{\phi} \in \mathcal{M}_{\mathrm{cl}}$ [5], and closed orbits are precisely those that contain a $D$ - flat point [9], therefore points in $\mathcal{M}_{\mathrm{cl}}$ are in one to one correspondence with D-flat points satisfying $d W=0$, i.e, classical supersymmetric vacua. 
The differential $\pi_{\phi_{0}}^{\prime}: T_{\phi_{0}} U^{W} \rightarrow T_{\hat{\phi}_{0}} \mathcal{M}_{\mathrm{cl}}$ of $\pi$ at $\phi_{0} \in U^{W}$ provides a linear map from the tangent of $U^{W}$ at $\phi_{0}$ to that of $\mathcal{M}_{\mathrm{cl}}$ at $\hat{\phi}_{0}=\pi\left(\phi_{0}\right)$. When $W=0, \mathcal{M}_{\mathrm{cl}}$ is just the algebraic subset of $V$ defined by the constraints among the $\hat{\phi}^{i}[$ [6]. As examples we introduce the two theories studied in this work:

Supersymmetric $Q C D$ : the gauge group is $G_{r}=S U(N)$, its complexification $G=S L(N, \mathbb{C})$. The matter fields $\phi$ are the quarks $Q^{i \alpha}, i=1, \ldots, N_{F}$ in the fundamental of $S U(N)$ and the antiquarks $\tilde{Q}_{\alpha j}, j=1, \ldots, N_{F}$ in the dual of $S U(N)$, so the dimension of $U$ is $d_{U}=2 N N_{F}$. A basic set of gauge invariant fields $\hat{\phi}^{i}$ is

$$
\begin{aligned}
M_{j}^{i} & =Q^{i \alpha} \tilde{Q}_{\alpha j} \\
B_{k_{1} \cdots k_{N_{D}}} & =Q^{i_{1} \alpha_{1}} Q^{i_{2} \alpha_{2}} \cdots Q^{i_{N} \alpha_{N}} \epsilon_{\alpha_{1} \alpha_{2} \cdots \alpha_{N}} \epsilon_{i_{1} i_{2} \cdots i_{N} k_{1} \cdots k_{N_{D}}} / N ! \\
\tilde{B}^{l_{1} \cdots l_{N_{D}}} & =\tilde{Q}_{\alpha_{1} j_{1}} \tilde{Q}_{\alpha_{2} j_{2}} \cdots \tilde{Q}_{\alpha_{N} j_{N}} \epsilon^{\alpha_{1} \alpha_{2} \cdots \alpha_{N}} \epsilon^{j_{1} j_{2} \cdots j_{N} l_{1} \cdots l_{N_{D}}} / N !
\end{aligned}
$$

where $N_{D}=N_{F}-N$; they span the vector space $V$ of dimension $N_{F}^{2}+2 N_{F} ! /\left(N_{D} ! N !\right)$. $W=0$, then $\mathcal{M}_{\mathrm{cl}}$ is the subset of $V$ defined by the algebraic constraints among the fields (113).

$S U(N)$ theories with matter in the adjoint: adding to the above theory a field $X$ in the adjoint (of $G=S L(N, \mathbb{C})$ ) and a tree level superpotential $W=p(\operatorname{tr} X), p$ a polynomial, we obtain the theories studied in [10]. We will concentrate on the case $W=\operatorname{tr} X^{3} / 3$. Computations are simpler if we drop the constraint $\operatorname{tr} X=0$, add a gauge singlet $T$ to the theory and replace the superpotential $W=\operatorname{tr} X^{3} / 3$ with

$$
W=\frac{1}{3} \operatorname{tr} X^{3}-\frac{T}{N} \operatorname{tr} X
$$

$T$ plays the role of a Lagrange multiplier. The equations $d W=0$ defining $U^{W}$ are $\operatorname{tr} X=0$ and $N X^{2}=T \mathbb{I}$. Note that, although $T$ and $\operatorname{tr} X^{k}$ are independent gauge invariants on $U$, only $T$ is independent on $U^{W}$, where $\operatorname{tr} X^{2 s+1}=0$ and $\operatorname{tr} X^{2 s}=T^{s} / N^{s-1}$, then a basic set of gauge invariants is 10

$$
\begin{aligned}
M_{j}^{i} & =Q^{i \alpha} \tilde{Q}_{\alpha j} \\
N_{j}^{i} & =Q^{i \alpha} X_{\alpha}^{\beta} \tilde{Q}_{\beta j}, \\
T( & \left.=X_{\beta}^{\alpha} X_{\alpha}^{\beta}\right), \\
B^{i_{1}, \ldots, i_{n_{1}} ; j_{1}, \ldots, j_{n_{2}}} & =Q^{i_{1} \alpha_{1}} Q^{i_{2} \alpha_{2}} \cdots Q^{i_{n_{1}} \alpha_{n_{1}}} X_{\gamma_{1}}^{\beta_{1}} \cdots X_{\gamma_{n_{2}}}^{\beta_{n_{2}}} Q^{j_{1} \gamma_{1}} \cdots Q^{j_{n_{2}} \gamma_{n_{2}}} \epsilon_{\alpha_{1} \cdots \alpha_{n_{1}} \beta_{1} \cdots \beta_{n_{2}}}, \\
\tilde{B}_{i_{1}, \ldots, i_{n_{1}} ; j_{1}, \ldots, j_{n_{2}}} & =\tilde{Q}_{\alpha_{1} i_{1}} \tilde{Q}_{\alpha_{2} i_{2}} \cdots \tilde{Q}_{\alpha_{n_{1} i_{n_{1}}}} X_{\beta_{1}}^{\gamma_{1}} \cdots X_{\beta_{n_{2}}}^{\gamma_{n_{2}}} \tilde{Q}_{\gamma_{1} j_{1}} \cdots \tilde{Q}_{\gamma_{n_{2} j_{n_{2}}} \epsilon^{\alpha_{1} \cdots \alpha_{n_{1}} \beta_{1} \cdots \beta_{n_{2}}},}
\end{aligned}
$$

where $n_{1}+n_{2}=N$. These fields span the vector space $V$.

We now list the results we need from [5], slightly generalized to the case $W \neq 0$, a detailed proof of these, together with a detailed description of $\mathcal{M}_{\mathrm{cl}}$ from an algebraic geometry approach can be found in [6].

Theorem I: (i) Assume $G$ is totally broken at $\phi_{0}$ and the orbit $G \phi_{0}$ is closed (equivalently, $G \phi_{0}$ contains a D-flat point [9, 6]), then $\pi_{\phi_{0}}^{\prime}: T_{\phi_{0}} U^{W} \rightarrow T_{\pi\left(\phi_{0}\right)} \mathcal{M}_{\mathrm{cl}}$ is onto. 
(ii) If also $U^{W}$ is irreducible 1 and $\phi_{0} \in U^{W}$ is smooth, then $\mathcal{M}_{\mathrm{cl}}$ is irreducible, $\hat{\phi}_{0}=\pi\left(\phi_{0}\right)$ is smooth, and $\operatorname{ker} \pi_{\phi_{0}}^{\prime}=\operatorname{Lie}(G) \phi_{0}$. In particular, $T_{\hat{\phi}_{0}} \mathcal{M}_{\mathrm{cl}}=\pi_{\phi_{0}}^{\prime}\left(T_{\phi_{0}} U^{W}\right)$ and $\operatorname{dim} \mathcal{M}_{\mathrm{cl}}=$ $\operatorname{rank} \pi_{\phi_{0}}^{\prime}=\operatorname{dim} U^{W}-\operatorname{dim} G$.

Theorem II: Let $\mathcal{M}_{\mathrm{cl}}$ be the classical moduli space of a supersymmetric gauge theory with gauge group $G$, superpotential $W$ and flavor symmetry $F$. It is assumed that the gauge theory has no gauge or gravitational anomalies, and the flavor symmetries have no gauge anomalies. Let $\hat{\phi}_{0} \in \mathcal{M}_{\mathrm{cl}}$ be a point in the classical moduli space. Assume there is a point $\phi_{0} \in U^{W}$ in the fiber $\pi^{-1}\left(\pi\left(\phi_{0}\right)\right)$ of $\hat{\phi}_{0}$ such that

(a) $G$ is completely broken at $\phi_{0}$.

(b) $\pi_{\phi_{0}}^{\prime}: T_{\phi_{0}} U^{W} \rightarrow T_{\hat{\phi}_{0}} \mathcal{M}_{\mathrm{cl}}$ is onto.

(c) $\operatorname{ker} \pi_{\phi_{0}}^{\prime}=\operatorname{Lie}(G) \phi_{0}$.

If a subgroup $F_{0} \subseteq F$ is unbroken at $\hat{\phi}_{0}$, then the 't Hooft consistency conditions for the $F_{0}^{3}$ flavor anomalies and the $F_{0}$ gravitational anomalies are satisfied, i.e, the anomalies computed in the $F_{0}$ invariant subspace $T_{\hat{\phi}_{0}} \mathcal{M}_{\mathrm{cl}}$ match the corresponding anomalies in $U$.

Corollary: If $\phi_{0}$ is a smooth point of the irreducible set $U^{W}$ that totally breaks $G$ and is D-flat (or has a closed $G$-orbit), then the anomalies of the flavor subgroup unbroken at $\hat{\phi}_{0}=\pi\left(\phi_{0}\right)$ match between $U$ and $T_{\hat{\phi}_{0}} \mathcal{M}_{\mathrm{cl}}$.

The matching of flavor anomalies of a theory and its dual can be explained as follows: the dual theory has complexified gauge group $G_{D}$, chiral configuration space space $U_{D}$, superpotential $W_{D}$ with critical points $U_{D}^{W_{D}}$. The $G_{D}$ invariant independent generators $\left(\hat{\phi}^{D}\right)^{i}\left(\phi^{D}\right)$ in $U_{D}^{W}$ span a vector space $V_{D}$. The global symmetry group of the dual theory is the same as the flavor group $F$ of the original theory. There is an isomorphism $I: V_{D} \rightarrow V$ (the span of $G$ invariant generators of the original theory) which commutes with the action of $F$, therefore both $\mathcal{M}_{\mathrm{cl}}$ and $\mathcal{M}_{\mathrm{cl}}^{D}$ can be thought embedded in the same vector space. In general, $\mathcal{M}_{\mathrm{cl}}$ and $\mathcal{M}_{\mathrm{cl}}^{D}$ are different. However, their intersection is nonempty and it is easy to find smooth points $\phi_{i} \in U^{W}, \phi_{i}^{D} \in U_{D}^{W_{D}}, i=1, \ldots, s$ satisfying the hypothesis of theorem I, therefore those of theorem II, such that $\pi\left(\phi_{i}\right)=\hat{\phi}_{i}=\pi_{D}\left(\phi_{i}^{D}\right)$. We can calculate the tangent spaces to $\mathcal{M}_{\mathrm{cl}}$ and $\mathcal{M}_{\mathrm{cl}}^{D}$ at $\hat{\phi}_{i}$ using theorem I, the superpotential $W_{D}$ is seen to be carefully chosen to satisfy $T_{\hat{\phi}_{i}} \mathcal{M}_{\mathrm{cl}}=\pi_{\phi_{i}}^{\prime}\left(T_{\phi_{i}} U^{W}\right)=\left(\pi_{D}\right)_{\phi_{i}^{D}}^{\prime}\left(T_{\phi_{i}^{D}} U_{D}^{W_{D}}\right)=T_{\hat{\phi}_{i}} \mathcal{M}_{\mathrm{cl}}^{D}$. In some restricted cases we may have $\mathcal{M}_{\mathrm{cl}}^{D}=\mathcal{M}_{\mathrm{cl}}$, then the above conditions are trivial. Denote $\mathcal{A}_{F}(X)$ the $F$ anomaly in the vector space $X$, and by $F_{i}$ the isotropy group of $\hat{\phi}_{i}$, i.e, the unbroken piece of $F$ at $\hat{\phi}_{i}$. Applying theorem II twice gives:

$$
\mathcal{A}_{F_{i}}(U)=\mathcal{A}_{F_{i}}\left(T_{\hat{\phi}_{i}} \mathcal{M}_{\mathrm{cl}}\right)=\mathcal{A}_{F_{i}}\left(T_{\hat{\phi}_{i}} \mathcal{M}_{\mathrm{cl}}^{D}\right)=\mathcal{A}_{F_{i}}\left(U_{D}\right)
$$

By choosing the points $\hat{\phi}_{i}$ appropriately, the matching of $U$ and $U_{D}$ anomalies for the full flavor group $F$ is implied by the matching of $F_{i}$ anomalies [6]. We remark that the argument

\footnotetext{
${ }^{1}$ This means that if the restriction to $U^{W}$ of the product of two polynomials in $U$ is zero, then the restriction of one of them must also be zero. The dimension of an irreducible algebraic set $X$ equals $\min _{x \in X} \operatorname{dim} T_{x} X$. The points of $X$ at which the tangent space has minimum dimension are said to be smooth [6, 8$]$.
} 
does not require the classical moduli spaces of both theories to be the same, but only to share the points $\hat{\phi}_{i}$, with the same tangent space at those points, and that these tangent spaces can be determined using theorem I, without even knowing the constraints that define $\mathcal{M}_{\mathrm{cl}}$.

Anomaly matching in dual SQCD theories: The origin of $\mathcal{M}_{\mathrm{cl}}$ in $N_{F}-2 \geq N \geq 2$ QCD theories displays the fully unbroken $S U\left(N_{F}\right)_{L} \times S U\left(N_{F}\right)_{R} \times U(1)_{B} \times R$ flavor symmetry; it is believed to correspond to a dual theory with $N_{F}$ flavors of quarks in the fundamental of the gauge group $S U\left(N_{D}\right), N_{D}=N_{F}-N, N_{F}$ flavors of antiquarks in the dual of the gauge group and additional gauge singlets $M_{j}^{i}$ [3]. Note that $N_{F}-2 \geq N_{D} \geq 2$ also. The transformation properties of the fields in both theories are summarized in the table below:

\begin{tabular}{|c|c|c|c|c|c|}
\hline & $G_{(D)}$ & $S U\left(N_{F}\right)_{L}$ & $S U\left(N_{F}\right)_{R}$ & $U(1)_{B}$ & $R$ \\
\hline$Q^{i \alpha}$ & $N$ & $N_{F}$ & - & 1 & $\frac{N_{F}-N}{N_{F}}$ \\
$\tilde{Q}_{j \alpha}$ & $\bar{N}$ & - & $\bar{N}_{F}$ & -1 & $\frac{N_{F}-N}{N_{F}}$ \\
\hline$q_{i}^{\alpha}$ & $N_{D}$ & $\bar{N}_{F}$ & - & $\frac{N}{N_{F}-N}$ & $\frac{N}{N_{F}}$ \\
$\tilde{q}_{\beta}^{j}$ & $\bar{N}_{D}$ & - & $N_{F}$ & $-\frac{N}{N_{F}-N}$ & $\frac{N}{N_{F}}$ \\
$M_{j}^{i}$ & - & $N_{F}$ & $\bar{N}_{F}$ & 0 & $2 \frac{N_{F}-N}{N_{F}}$ \\
\hline
\end{tabular}

The motivation behind the flavor transformation properties of the fields in the dual theory is that the gauge invariant polynomials

$$
\begin{aligned}
B_{i_{1} \cdots i_{N_{D}}} & =q_{i_{1}}^{\alpha_{1}} q_{i_{2}}^{\alpha_{2}} \cdots q_{i_{N}}^{\alpha_{N_{D}}} \epsilon_{\alpha_{1} \alpha_{2} \cdots \alpha_{N_{D}}} \\
\tilde{B}^{j_{1} \cdots j_{N_{D}}} & =\tilde{q}_{\alpha_{1}}^{j_{1}} \tilde{q}_{\alpha_{2}}^{j_{2}} \cdots \tilde{q}_{\alpha_{N_{D}}}^{j_{N_{D}}} \epsilon^{\alpha_{1} \alpha_{2} \cdots \alpha_{N_{D}}},
\end{aligned}
$$

can be identified with the fields (2, 3) of the original theory. The identification of (1) with the gauge singlets $M_{j}^{i}$ of the dual theory completes the isomorphism $I: V_{D} \rightarrow V$. The gauge invariants $q_{i}^{\alpha} \tilde{q}_{\alpha}^{j}$ are trivial on $U^{W_{D}}$, as $W_{D}=M_{j}^{i} q_{i}^{\alpha} \tilde{q}_{\alpha}^{j}$. We can show flavor anomaly matching for these theories using only two points, $\phi_{1}$ and $\phi_{2}$, in the above argument. $\phi_{1}$ has coordinates

$$
Q^{i \alpha}=\left\{\begin{array}{ll}
m \delta^{i \alpha} & i \leq N \\
0 & i>N
\end{array}, \quad \tilde{Q}_{j \alpha}=0\right.
$$

Note that $G \phi_{1}=\left\{\left(Q^{i \alpha}, \tilde{Q}_{\beta j}\right) \mid \operatorname{det}_{i \leq \alpha} Q=m^{N}, \tilde{Q}_{\beta j}=Q^{i \alpha}=0, i>\alpha\right\}$ is a closed set, and that $G$ is totally broken at $\phi_{1}$, then theorems I and II apply at $\phi_{1}$. The point $\pi\left(\phi_{1}\right)=\hat{\phi}_{1}$ in the IR theory is described by gauge invariant meson and baryon fields,

$$
M_{j}^{i}=0, \quad \tilde{B}^{j_{1} \cdots j_{N_{D}}}=0, \quad B_{i_{1} \cdots i_{N_{D}}}=m^{N} \epsilon_{12 \ldots N_{c} i_{1} \cdots i_{N_{D}}} .
$$

The unbroken flavor group at $\hat{\phi}_{1}$ is $F_{1}=S U(N)_{L} \times S U\left(N_{D}\right)_{L} \times S U\left(N_{F}\right)_{R} \times U(1)_{B}^{\prime} \times U(1)_{R}^{\prime}$, where (the Lie algebra of) $U(1)_{B}^{\prime} \times R^{\prime}$ is a linear combination of the original baryon and $R$ symmetry generators and generators of $S U\left(N_{F}\right)_{L}$. Under these unbroken symmetries, the fields transform as 


\begin{tabular}{|c|c|c|c|c|c|}
\hline & $S U(N)_{L}$ & $S U\left(N_{D}\right)_{L}$ & $S U\left(N_{F}\right)_{R}$ & $U(1)_{B}^{\prime}$ & $U(1)_{R}^{\prime}$ \\
\hline$Q^{i \alpha}, i \leq N$ & $N$ & - & - & 0 & 0 \\
$Q^{i \alpha}, i>N$ & - & $N_{D}$ & - & $-N_{F}$ & $\left(2 N_{F}-2 N\right) /\left(2 N_{F}-N\right)$ \\
$\tilde{Q}_{j \alpha}$ & - & - & $\bar{N}_{F}$ & $N_{D}$ & $\left(2 N_{F}-2 N\right) /\left(2 N_{F}-N\right)$ \\
\hline
\end{tabular}

A natural choice for $\phi_{1}^{D}$ in the dual theory is

$$
q_{i}^{\alpha}=\left\{\begin{array}{ll}
m \delta_{i-N}^{\alpha} & i>N \\
0 & i \leq N
\end{array}, \quad \tilde{q}_{\alpha}^{j}=0, \quad M_{j}^{i}=0\right.
$$

Although the classical moduli spaces of these theories are different ( $M_{j}^{i}$ can be arbitrary in the dual theory, whereas rank $M_{j}^{i} \leq N$ in the original theory), we can use theorem I to check that, thanks to the superpotential $W_{D}$ in the dual theory, the tangents at the shared point (13) agree, as $\pi_{\phi_{1}}^{\prime}(U)=\pi_{\phi_{1}^{D}}^{\prime}\left(T_{\phi_{1}^{D}} U_{D}^{W}\right)$. This is the span of $\delta M_{j}^{i}, i \leq N$ and the $\delta B_{i_{1}, \ldots, i_{N_{D}}}$ with at most one of the $i_{k}$ less than or equal to $N$. Note that $T_{\phi_{1}^{D}} U_{D}^{W}=\left.\operatorname{ker}\left(\partial W /\left(\partial\left(\phi^{D}\right)^{i} \partial\left(\phi^{D}\right)^{j}\right)\right)\right|_{\phi_{1}^{D}}=\operatorname{span}\left(\delta M_{j}^{i}(i \leq N), \delta q_{i}^{\alpha}\right)$, and that ker $\pi_{\phi_{1}^{D}}^{\prime}$ is the subspace $\left.T_{\phi_{1}^{D}} U_{D}^{W} \cap \operatorname{ker}\left(\partial \hat{\phi}^{i} / \partial\left(\phi^{D}\right)^{j}\right)\right)\left.\right|_{\phi_{1}^{D}}=\operatorname{Lie}(G) \phi_{1}^{D}$, as anticipated by Theorem I. \& The point $\phi_{2}$ is taken to be "symmetric" to $\phi_{1}$ (i.e., with the roles of $Q$ and $\tilde{Q}$ exchanged). The matching of the full flavor group anomalies then follows from the matching of $F_{1}$ and $F_{2}$ anomalies [6].

Anomaly matching in dual $S U(N)$ theories with adjoint matter: As in the QCD case, not every point in $\mathcal{M}_{\mathrm{cl}}$ describes correctly the massless particle spectrum in the IR, but only those predicted by theorem II. A dual theory based on the gauge group $S U\left(N_{D}^{\prime}\right), N_{D}^{\prime}=$ $2 N_{F}-N, N_{F}$ flavors of fundamentals and conjugate fields, an adjoint and additional singlets is believed to describe the vacuum at the origin, where the full flavor symmetry group $S U\left(N_{F}\right)_{L} \times S U\left(N_{F}\right)_{R} \times U(1)_{B} \times R$ is unbroken. The field content and transformation properties for both theories are summarized in the table below [10]

\begin{tabular}{|c|c|c|c|c|c|}
\hline & $G_{(D)}$ & $S U\left(N_{F}\right)_{L}$ & $S U\left(N_{F}\right)_{R}$ & $U(1)_{B}$ & $R$ \\
\hline$Q^{i \alpha}$ & $N$ & $N_{F}$ & - & 1 & $1-\frac{2 N}{3 N_{F}}$ \\
$\tilde{Q}_{j \alpha}$ & $\bar{N}$ & - & $\bar{N}_{F}$ & -1 & $1-\frac{2 N}{3 N_{F}}$ \\
$X$ & $\mathrm{Adj}$ & - & - & 0 & $\frac{2}{3}$ \\
$T$ & - & - & - & 0 & $\frac{4}{3}$ \\
\hline$q_{i}^{\alpha}$ & $N_{D}^{\prime}$ & $\bar{N}_{F}$ & - & $\frac{N}{N_{D}^{\prime}}$ & $1-\frac{2 N_{D}^{\prime}}{3 N_{F}}$ \\
$\tilde{q}_{\beta}^{j}$ & $\bar{N}_{D}^{\prime}$ & - & $N_{F}$ & $-\frac{N}{N_{D}^{\prime}}$ & $1-\frac{2 N_{D}^{\prime}}{3 N_{F}}$ \\
$Y$ & $\mathrm{Adj}$ & - & - & 0 & $\frac{2}{3}$ \\
$M_{j}^{i}$ & - & $N_{F}$ & $\bar{N}_{F}$ & 0 & $2-\frac{4 N}{3 N_{F}}$ \\
$N_{j}^{i}$ & - & $N_{F}$ & $\bar{N}_{F}$ & 0 & $\frac{8 N_{F}-4 N}{3 N_{F}}$ \\
$T$ & - & - & - & 0 & $\frac{4}{3}$ \\
\hline
\end{tabular}

\footnotetext{
${ }^{2}$ When $W \neq 0$ it may be easier to check the equality ker $\pi_{\phi_{0}}^{\prime}=\operatorname{Lie}(G) \phi_{0}$ by using ker $\pi_{\phi_{0}}^{\prime}=$ $\left.T_{\phi_{0}} U^{W} \cap \operatorname{ker}\left(\partial \hat{\phi}^{i} / \partial \phi^{j}\right)\right|_{\phi_{0}}$, rather than proving that $U^{W}$ is irreducible and $\phi_{0}$ smooth to apply theorem I.
} 
We can identify the invariant fields [8,9] with

$$
\begin{aligned}
B^{i_{1}, \ldots, i_{n_{1}} ; j_{1}, \ldots, j_{n_{2}}}= & \frac{1}{N !} \epsilon^{i_{1}, \ldots, i_{n_{1}}, l_{1}, \ldots, l_{n_{2}}} \epsilon^{j_{1}, \ldots, j_{n_{2}}, k_{1}, \ldots, k_{n_{1}}} \\
& q_{k_{1}}^{\alpha_{1}} q_{k_{2}}^{\alpha_{2}} \cdots q_{k_{n_{1}}}^{\alpha_{n_{1} D}} Y_{\gamma_{1}}^{\beta_{1}} \cdots Y_{\gamma_{n} D}^{\beta_{n_{2} D}} q_{l_{1}}^{\gamma_{1}} \cdots q_{l_{n_{2}}}^{\gamma_{n_{2} D}} \epsilon_{\alpha_{1} \cdots \alpha_{n_{1}} \beta_{1} \cdots \beta_{n_{2}}}, \\
\tilde{B}_{i_{1}, \ldots, i_{n_{1}} ; j_{1}, \ldots, j_{n_{2}}}= & \frac{1}{N !} \epsilon_{i_{1}, \ldots, i_{n_{1}} ; l_{1}, \ldots, l_{n_{2}}} \epsilon_{j_{1}, \ldots, j_{n} ; k_{1}, \ldots, k_{n_{1}}} \\
& \tilde{q}_{\alpha_{1}}^{k_{1}} \tilde{q}_{\alpha_{2}}^{k_{2}} \cdots \tilde{q}_{\alpha_{n_{1} D} D}^{k_{n_{1} D}} Y_{\beta_{1}}^{\gamma_{1}} \cdots Y_{\beta_{n_{2} D}}^{\gamma_{n_{2} D}} \tilde{q}_{\gamma_{1}}^{l_{1}} \cdots \tilde{q}_{\gamma_{n_{2} D}}^{j_{n_{2}}} \epsilon^{\alpha_{1} \cdots \alpha_{n_{1} D} \beta_{1} \cdots \beta_{n_{2} D}},
\end{aligned}
$$

where $n_{1}^{D}=N_{F}-n_{2}, n_{2}^{D}=N_{F}-n_{1}$. The identification of the invariants (5), (6) and (匹) with the singlets $M_{j}^{i}, N_{j}^{i}$ and $T$ of the dual theory completes the map $I: V_{D} \rightarrow V$. A superpotential

$$
W_{D}=\frac{1}{3} \operatorname{tr} Y^{3}+M_{j}^{i} \tilde{q}_{\alpha}^{j} q_{i}^{\alpha}+N_{j}^{i} \tilde{q}_{\alpha}^{j} Y_{\beta}^{\alpha} q_{i}^{\beta}-\frac{T}{N} \operatorname{tr} Y^{2}
$$

is added to the dual theory, then the gauge invariants $\tilde{q}_{\alpha}^{j} q_{i}^{\alpha}$ and $\tilde{q}_{\alpha}^{i} Y_{\beta}^{\alpha} q_{i}^{\beta}$ need not be considered, as they are trivial on $U_{D}^{W_{D}}$. Also, $\operatorname{tr} Y^{2}=T$ on $U_{D}^{W_{D}}$. Note that $N+s=N_{F}=N_{D}-s$. As duality is an involutive operation we can restrict ourselves to the study of the $s \leq 0$ case. To understand anomaly matching we can restrict further to the self dual case $s=0$, as we can flow to the other cases by adding a mass term to the "electric" theory to decouple a flavor. Duality is compatible with this flow, which also preserves anomaly matching [5,6]. The motivation behind considering the $s=0$ case is that more similarities between the classical chiral rings of the original and dual theories are to be expected in this case [10]. Finally, assume for simplicity $N=2 n$ (Higgs effect allows us to flow to the odd $N$ case) and consider the point $\phi_{1}$ of coordinates

$$
X=m\left(\begin{array}{ll}
0 & 1 \\
0 & 0
\end{array}\right), \quad Q=m\left(\begin{array}{cc}
0 & \sqrt{2} \\
0 & 0
\end{array}\right), \quad \tilde{Q}=0 .
$$

In the above matrix notation upper or left indices label rows, and $X, Q$ and $\tilde{Q}$ are broken up in square matrices of size $n . \quad \phi_{1}$ is a smooth point in the irreducible set $U^{W}$ which breaks $G=S L(n, \mathbb{C})$ completely and is $D$ flat, then theorems I and II apply (see however footnote 3$)$. The only nonzero coordinates of $\hat{\phi}_{1}=\pi\left(\phi_{1}\right)$ are $B^{i_{1}, \ldots, i_{n} ; j_{1}, \ldots, j_{n}}=$ $2^{n} m^{3 n} \epsilon^{i_{1}, \ldots, i_{n}, n+1, \ldots, 2 n} \epsilon^{j_{1}, \ldots, j_{n}, n+1, \ldots, 2 n}$. We choose $\phi_{1}^{D}$ to be the point with coordinates

$$
Y=\alpha m\left(\begin{array}{ll}
0 & 0 \\
1 & 0
\end{array}\right), \quad q=\alpha m\left(\begin{array}{cc}
0 & \sqrt{2} \\
0 & 0
\end{array}\right), \quad \tilde{q}=M=N=0
$$

where $\alpha^{3 n}=-N ! /(n !)^{4}$. It is straightforward to verify that $\phi_{1}^{D}$ is in $U_{D}^{W_{D}}$ and satisfies the hypothesis of the matching theorem. The tangents $T_{\hat{\phi}_{1}} \mathcal{M}_{\mathrm{cl}}$ and $T_{\hat{\phi}_{1}} \mathcal{M}_{\mathrm{cl}}^{D}$ both equal the span of $\delta M_{j}^{i}, i \leq n$, and $\delta N_{j}^{i}, i \leq n, \delta T$, and the fields $\delta B^{i_{1}, \ldots, i_{n}, j_{1}, \ldots, j_{n}}$ with at most one index bigger than $n$ (the nonzero fields $\delta B^{i_{1}, \ldots, i_{n_{1}}, j_{1}, \ldots, j_{n_{2}}}$ with $n_{1}=n \pm 1$ and at most one index bigger than $n$ are linearly dependent from these). The vacuum $\hat{\phi}_{1}$ breaks $F$ to $F_{1}=S U(n)_{L} \times S U(n)_{L} \times S U(N)_{R} \times U(1)_{B}^{\prime} \times R^{\prime}$, with $U(1)_{B}^{\prime} \times R^{\prime}$ a combination of $U(1)_{B} \times R$ 
and $S U(N)_{L}$. We can take flavor rotated versions of $(18,19)$, or just the "symmetric" point with $Q^{i \alpha}=0$ to complete the proof of anomaly matching.

Our results allow the prediction of the dimension of the dual group $G_{D}$. Assume the matter content and superpotential of the dual theory are obtained, e.g, from the rules in [11]), up to the value of $N_{D}$. Our anomaly matching mechanism implies

$$
\operatorname{dim} \mathcal{M}_{\mathrm{cl}}=\operatorname{dim} \mathcal{M}_{\mathrm{cl}}^{D} \text {. }
$$

Any two (unrelated) points $\phi_{0} \in U^{W}$ and $\phi_{0}^{D} \in U_{D}^{W_{D}}$ satisfying the hypothesis of Theorem I (d) can be used to calculate these dimensions

$$
\operatorname{dim} \mathcal{M}_{\mathrm{cl}}=\operatorname{rank} \pi_{\phi_{0}}^{\prime}=\operatorname{dim} T_{\phi_{0}} U^{W}-\operatorname{dim} G=\operatorname{dim} U^{W}-\operatorname{dim} G,
$$

and analogously for $\mathcal{M}_{\mathrm{cl}}^{D}$. This calculation can be done even before checking any connection between $V$ and $V_{D}$. In the QCD example, using the points of Eqs. (12, 14), we obtain $\operatorname{dim} U=2 N N_{F}, \operatorname{dim} U^{W}=\operatorname{dim} T_{\phi_{0}} U^{W}$. The latter is spanned by the $N_{D} N_{F} \delta q_{i}^{\alpha}$ and the $\left(N_{F}-N_{D}\right) N_{F} \delta M_{j}^{i}, i \leq N_{F}-N_{D}$, then (20,21) give the following equation on the indeterminate $N_{D}$ :

$$
2 N N_{F}-\left(N^{2}-1\right)=N_{D} N_{F}+\left(N_{F}-N_{D}\right) N_{F}-\left(N_{D}^{2}-1\right) .
$$

The solutions of this equation are $N_{D}= \pm\left(N_{F}-N\right)$. For the theory with matter in the adjoint, we analyze the case $N$ even and assume $N_{D}$ is also even, with $N_{F}>N / 2, N_{D} / 2$. To calculate dimensions we use suitable generalizations of Eqs. (18,19) to the $N_{F} \neq N$ case, where now the lower $Q^{i \alpha}$ blocks are $\left(N_{f}-N / 2\right) \times N / 2$ matrices and the left hand side $q_{j}^{\alpha}$ blocks are $\left(N_{D} / 2\right) \times\left(N_{F}-N_{D} / 2\right)$ matrices. The tangent $T_{\phi_{0}} U^{W}$ is spanned by $N^{2} / 2$ fields $\delta X_{\beta}^{\alpha}, \delta T$, and the unconstrained $\delta Q^{i \alpha}$ fields, so we get

$$
\operatorname{dim} \mathcal{M}_{\mathrm{cl}}=\left(\frac{N^{2}}{2}\right)+1+2 N N_{F}-\left(N^{2}-1\right) .
$$

The tangent $T_{\phi_{0}^{D}} U_{D}^{W_{D}}$ is spanned by $N_{D}^{2} / 2$ fields $\delta Y_{\beta}^{\alpha}, \delta T$, the unconstrained $\delta Q^{i \alpha}$, and the $\delta M_{j}^{i}$ and $\delta N_{j}^{i}$ with $i \leq N_{F}-N_{D} / 2$, therefore

$$
\operatorname{dim} \mathcal{M}_{\mathrm{cl}}^{D}=\left(\frac{N_{D}^{2}}{2}\right)+1+N_{D} N_{F}+2\left(N_{F}-\frac{N_{D}}{2}\right) N_{F}-\left(N_{D}^{2}-1\right) .
$$

Equating (22) and (23) we obtain $N_{D}= \pm\left(2 N_{F}-N\right)$, as expected.

Understanding flavor anomaly matching for other pairs of dual theories like those involving $S O(N)$ and $S P(2 N)$ gauge groups requires stronger versions of theorem II which are currently under study, their treatment seems to be analogous to the simpler cases presented here. The studied examples suggest that the satisfaction of ' $t$ Hooft's consistency conditions is to be expected from the similarities of the classical moduli spaces of dual theories, and does not constitute an independent test on the duality hypothesis.

I would like to thank K. Intriligator, A. Manohar, E. Poppitz and W. Skiba for useful discussions and comments on the manuscript. This work was supported in part by a Department of Energy grant DOE-FG03-97ER40546. 


\section{REFERENCES}

[1] N. Seiberg, Phys. Rev. D49 (1994) 6857, K. Intriligator, R.G. Leigh, N. Seiberg, Phys. Rev. D50 (1994) 1092, K. Intriligator and N. Seiberg, Nucl. Phys. B444 (1995) 125. For a more detailed list of references, see K. Intriligator and N. Seiberg hep-th/9509066 and M.E. Peskin hep-th/9702014.

[2] G. 't Hooft, Naturalness, Chiral Symmetry and Chiral Symmetry Breaking, Lectures given at the Cargèse Summer Institute, Cargèse, France, 1979.

[3] N. Seiberg, Nucl. Phys. B435 (1995), 129.

[4] I. Affleck, M. Dine, and N. Seiberg, Nucl. Phys. B241 (1984) 493.

[5] G. Dotti and A.V. Manohar, hep-th 9706075, to appear in Phys. Lett. B.

[6] G. Dotti and A.V. Manohar, UCSD/PTH 97-14 (in preparation).

[7] C. Csaki, M. Schmaltz and W. Skiba, Phys. Rev. D55 (1997) 7840.

[8] R. Goodman and N.R. Wallach, Representation and Invariants of the Classical Groups, (Cambridge University Press, Cambridge, 1997). Appendix A and Chapter XI.

[9] M.A. Luty and W. Taylor IV, Phys. Rev. D53 (1996) 3399.

[10] D. Kutasov, Phys. Lett. B351 (1995) 230, D. Kutasov and A. Schwimmer, Phys Lett. B354 (1995), 315, D. Kutasov, A. Schwimmer, and N. Seiberg, Nucl. Phys. B459 (1996), 455.

[11] N. Evans and M. Schmaltz, Phys. Rev. D55 (1997) 3776. 